\title{
Study of the non-parasitic stage in Ixodes ricinus after co-feeding with Dermacentor reticulatus in three infestations
}

\author{
Katarzyna Bartosik', Alicja Buczek ${ }^{1}$, Adam Borzęcki², Dorota Kulina ${ }^{3}$ \\ ${ }^{1}$ Chair and Department of Biology and Parasitology, Medical University, Lublin, Poland \\ ${ }^{2}$ Non-Public Health Care Center Med-Laser, Lublin, Poland \\ ${ }^{3}$ Department of Basic Nursing and Medical Teaching, Medical University, Lublin, Poland
}

Bartosik K, Buczek A, Borzęcki A, Kulina D. Study of the non-parasitic stage in Ixodes ricinus after co-feeding with Dermacentor reticulatus in three infestations. Ann Agric Environ Med. 2017; 24(1): 90-95. doi: 10.5604/12321966.1234005

\begin{abstract}
I Abstract
It was proved that transmission of some pathogens may occur between infected and uninfected ticks co-feeding on a host in the absence of systemic infection. The effect was studied of co-feeding of two different tick species /xodes ricinus and Dermacentor reticulatus on the course of the non-parasitic stage. Species chosen for the experiment may share their habitats and co-infest animals in nature. In the course of the study the process of egg maturation, oviposition and larval hatching were investigated. In order to estimate if co-feeding influence can counteract host immunity, three subsequent infestations of rabbits were analyzed. Mono-specific groups values of such a parameters as egg amount, number of eggs per $1 \mathrm{mg}$ of female engorgement weight, female oviposition weight loss, hatching success were higher in inter-specific groups were compared. The results indicate that co-feeding with other tick species may partially reduce the influence of host resistance in subsequent infestation.
\end{abstract}

\section{Key words}

Ixodes ricinus; Dermacentor reticulatus; co-feeding ticks; repeated infestations; tick reproduction.

\section{INTRODUCTION}

The humidity and temperature preferences of ticks - Ixodes ricinus (Prostriata group) and Dermacentor reticulatus (Metastriata group) - indicate the possibility of them living in the same habitats [1] (corroborated also by the authors' field observations), and the broad host spectrum implies their ability to infest the same animals [2,3] and humans [4]. However, little is known about the mutual interactions between the two species in nature and on the host, and especially about their host-seeking behaviour, location of individuals of the opposite gender for copulation, and about their feeding behaviour. Co-feeding of ticks is a subject of increasing interest as transmission of some pathogens may occur between infected and uninfected ticks while feeding on a host in the absence of systemic infection [5,6]. Aggregation of ticks at one site during co-feeding has been shown not only to facilitate tick-borne pathogen transmission, but also increase the feeding success of the parasite [7]. Due to repeated tick invasions, the host gradually develops a resistance and its immunity significantly decrease ticks vitality. Acquired resistance in the host disturbs feeding, egg laying and hatching $[8,9]$. The world literature fails to provide data about the mutual interactions between I. ricinus and $D$. reticulatus in their parasitic activity on the same host, and about the resultant changes in their biology.

Address for correspondence: Katarzyna Bartosik, Cgair and Department of Biology and Parasitology, Medical University, Lublin, Poland

E-mail: katarzyna.bartosik@umlub.pl

Received: 31 March 2016; accepted: 18 May 2016; first published on January 2017

\section{OBJECTIVE}

The aim of this study was to determine whether the cofeeding of two species on the same host influences their non-parasitic stage of the life cycle, i.e. the process of egg maturation and oviposition and larval hatching. The course of subsequent infestations I, II and III of the same host by various tick species, and factors that induce disturbance of the non-parasitic stage of the developmental cycle were also studied. The range of interactions between I. ricinus and $D$. reticulatus co-occurring on the same host and of the biological effects of the intra- and interspecies interactions between both tick species were additionally assayed.

\section{MATERIALS AND METHOD}

Host. Tick-naive New Zealand albino rabbits (Oryctolagus cuniculus) were used in the study. When the experiments were commenced, the 6-month-old females had an average body weight of $3-3.5 \mathrm{~kg}$. Rabbits were given ad libitum access to food and water and kept under standard laboratory conditions. All experiments were carried out in accordance with local institutional ethical guidelines about animals experimentation, and every effort was made to minimize the number of animals used and their suffering.

The first infestation was performed on 8 rabbits. Six of them were used for studying a concurrent infestation with mixed groups of ticks. Two rabbits were subjected to a control infestation with mono-specific group of I. ricinus ticks.

Collection and breeding of ticks. Un-engorged D. reticulatus and $I$. ricinus individuals were collected in the same habitats during the spring peak of their activity. The ticks 
were collected with the commonly used flagging method, which involves sweeping the grass, short shrubs and ground surfaces with a $1 \mathrm{~m}^{2}$ white flannel cloth. The specimens were placed in glass containers, in which the conditions were similar to those in tick habitats, i.e. $80-90 \%$ humidity and $25^{\circ} \mathrm{C}$ temperature. The experiments were conducted on 160 females and 80 males of $I$. ricinus and 120 females and 60 males of $D$. reticulatus.

The dependencies between the tick species in the parasitic stage of the life cycle were investigated in the following experimental groups:

- infestations with one tick species (mono-specific infestations) - 20 females and 10 males of I. ricinus;

- concurrent inter-specific infestations comprising 20 females and 10 males of $I$. ricinus, and 20 females and 10 males of $D$. reticulatus,

The above-mentioned scheme was also applied in the experiments performed after tick infestations II and III, which commenced 3 days after the ticks ceased feeding on the previously infested rabbits. The effect of host resistance on the development of ticks in the parasitic and non-parasitic stage of the life cycle was assessed on the basis of biological traits, such as feeding, egg-laying, and the larval development and hatching.

Study of the biology of ticks in the non-parasitic stage. After drop-off, engorged I. ricinus females were weighed with an accuracy of $0.001 \mathrm{~g}$ and transferred to separate breeding chambers. The breeding chambers with engorged females from the various experimental groups were kept under constant conditions: temperature of $30^{\circ} \mathrm{C}$ and approximate humidity $100 \%$ maintained with distilled water at 24:0 nightday photoperiod. The experiments were assessed at the same time daily; special attention was paid to the first egg-laying. The mass of all the eggs produced by individual females as well as the females' body mass after the egg-laying process were measured after the last egg-laying.

The laid eggs of $I$. ricinus were kept at the temperature of $30^{\circ} \mathrm{C}$ and $100 \%$ humidity. The eggs were observed daily under a stereoscopic microscope at magnification 40x10 until embryogenesis was completed and larvae hatched.

Parameters of the non-parasitic stage. In all the study groups, parameters of the non-parasitic stage of the life cycle in $I$. ricinus were determined, i.e. the parameters characteristic for the preoviposition and oviposition periods and those associated with larval hatching.

The preoviposition period (PP), i.e. the period of egg maturation, is the period between cessation of feeding and the first egg-laying (expressed in days, with an accuracy of 1 day), egg mass weight (EMW) - the mass of all the eggs laid by a female (expressed in grams, with an accuracy of $0.001 \mathrm{~g}$ ), egg amount (EA) - the number of eggs laid by one female, female oviposition weight loss (FOWL) - the index of weight loss in females after laying eggs (expressed as percent, with an accuracy of $0.1 \%$ ), egg laying frequency (ELF) - the percentage of the study females which laid eggs (expressed as percent, with an accuracy of $1 \%$ ), hatching success (HS) - demonstrates the percentage of the laid eggs from which larvae hatched (expressed as percent, with an accuracy of $0.1 \%)$.
Statistical methods in data analysis. Parameters of the developmental cycle of $I$. ricinus in 3 mono-specific and 3 inter-specific repeated infestations were analysed. They were characterised with the use of the arithmetic mean $(M)$, which reflects the average level and the standard deviation $(S D)$ which determines the range of measurement value dispersion around the arithmetic mean.

The Mann-Whitney U test was employed for determination of significant differences in the parameter values between the first and second, the first and third and the second and third infestations in the mono-specific groups, and then in the inter-specific groups. The same test was subsequently used in order to check whether there were significant differences in the parameter values between the mono-specific and interspecific groups in the same infestations. The Tables present the $U$ test statistics values and the critical $p$ significance level, i.e. the lowest - at particular probe results - significance level, at which the null hypothesis may be rejected. The significance level was set at $\alpha=0.05$, thus the verification decisions are as follows: if $p>0.05$, there are no grounds for rejecting the null hypothesis of equality of the parameters in both groups; in contrast, if $p \leq 0.05$, the null hypothesis should be rejected in favour of the alternative hypothesis, which implies the existence of significant differences between the two groups.

The calculations were performed and the graphs prepared with Statistica 5 PL and Microsoft Excel XP programmes.

\section{RESULTS}

Egg maturation and oviposition in I. ricinus after co-feeding with $D$. reticulatus in the 3 infestations.

1) Preoviposition period (PP). In I. ricinus females feeding on the rabbits in the inter-specific group in infestation I, the oviposition started as early as 5-12 days (mean $8.46 \pm$ 1.502) after cessation of feeding at the temperature of $30^{\circ} \mathrm{C}$ and $100 \%$ RH (Tab. 1). Similar to the mono-specific groups, the length of the preoviposition period decreased in the reinfestations, on average, was $7.56 \pm 0.85$ days in infestation II and $7.23 \pm 0.81$ days in III (Tab. 1, 2). The differences observed in the length of the egg maturation period in the successive infestations are statistically significant for infestations I and II, as well as I and III ( $U=265.50 ; p=0.006$ and $U=207$; $\mathrm{p}=0.000$, respectively). The length of the preoviposition period in the inter-specific groups did not differ significantly in comparison with the mono-specific groups (Tab. 1, 2).

2) Egg mass weight (EMW). After infestation I, the I. ricinus females co-feeding on the rabbits with $D$. reticulatus laid eggs were characterised by the highest weight (mean $0.18 \pm 0.02 \mathrm{~g}$ ). Similar to the mono-specific groups, there was a decline in the weight of eggs laid by females feeding in the subsequent infestations, with the lowest weight value after infestation III (mean $0.06 \pm 0.01$ ) (Tab. 1,2). The weight of the eggs laid by I. ricinus in the study groups differed significantly after the successive infestations. The values determined by the MannWhitney $U$ test were $U=60.00 ; p=0.000$ for infestations I and II, $U=0.00 ; p=0.000$ for infestations II and III, and $U=175.50$; $\mathrm{p}=0.000$ for infestations I and III. The weight of the eggs laid by the females after co-feeding in the inter-specific groups did not differ significantly from that in the mono-specific groups (Tab. 1). 
Table 1. Egg maturation and oviposition parameters in Ixodes ricinus in 3 infestations

\begin{tabular}{|c|c|c|c|c|c|}
\hline Parameter & $\begin{array}{l}\text { Successive } \\
\text { infestations }\end{array}$ & $\min$ & $\max$ & M & SD \\
\hline \multirow{3}{*}{ Preoviposition period (PP) (days) } & I & 7 & 9 & 8.00 & 0.37 \\
\hline & II & 7 & 9 & 7.50 & 0.57 \\
\hline & III & 7 & 8 & 7.13 & 0.35 \\
\hline \multirow{3}{*}{ Egg mass weight (EMW) (g) } & I & 0.12 & 0.23 & 0.18 & 0.02 \\
\hline & II & 0.06 & 0.20 & 0.11 & 0.02 \\
\hline & III & 0.04 & 0.09 & 0.06 & 0.01 \\
\hline \multirow{3}{*}{ Egg Amount (EA) } & I & 993 & 1238 & 1103 & 124 \\
\hline & II & 980 & 1022 & 1003 & 21 \\
\hline & III & 657 & 712 & 676 & 31 \\
\hline \multirow{3}{*}{$\begin{array}{l}\text { Female oviposition weight loss } \\
\text { (FOWL) }\end{array}$} & I & 50.90 & 73.33 & 64.58 & 5.23 \\
\hline & II & 35.66 & 66.79 & 55.00 & 8.08 \\
\hline & III & 46.36 & 73.51 & 60.01 & 7.22 \\
\hline \multirow{3}{*}{ Egg laying frequency (ELF) } & I & \multicolumn{4}{|c|}{100} \\
\hline & ॥ & \multicolumn{4}{|c|}{100} \\
\hline & III & \multicolumn{4}{|c|}{73} \\
\hline
\end{tabular}

$\mathrm{M}$ - arithmetic mean; SD - standard deviation

Table 2. Egg maturation and oviposition parameters in /xodes ricinus after co-feeding with Dermacentor reticulatus in 3 infestations

\begin{tabular}{|c|c|c|c|c|c|c|c|}
\hline Parameter & $\begin{array}{l}\text { Successive } \\
\text { infestations }\end{array}$ & $\min$ & $\max$ & M & SD & $\begin{array}{c}\text { Test } \\
\mathrm{U}\end{array}$ & $\mathrm{p}$ \\
\hline \multirow{3}{*}{$\begin{array}{l}\text { Preoviposition } \\
\text { period (PP) (days) }\end{array}$} & 1 & 5 & 12 & 8.46 & 1.50 & 375.0 & 0,267 \\
\hline & II & 6 & 10 & 7.56 & 0.85 & 449.0 & 0,988 \\
\hline & III & 6 & 9 & 7.23 & 0.81 & 309.5 & 0,704 \\
\hline \multirow{3}{*}{$\begin{array}{l}\text { Egg mass weight } \\
(\text { EMW) }(g)\end{array}$} & 1 & 0.12 & 0.23 & 0.18 & 0.02 & 334.5 & 0,087 \\
\hline & II & 0.02 & 0.20 & 0.10 & 0.04 & 393.0 & 0,399 \\
\hline & III & 0.04 & 0.08 & 0.06 & 0.01 & 240.0 & 0,095 \\
\hline \multirow{3}{*}{ Egg Amount (EA) } & 1 & 1502 & 1805 & 1637 & 154 & 0.0 & 0,049 \\
\hline & II & 1076 & 1392 & 1230 & 158 & 0.0 & 0,049 \\
\hline & III & 778 & 1003 & 872 & 117 & 0.0 & 0,049 \\
\hline \multirow{3}{*}{$\begin{array}{l}\text { Female oviposition } \\
\text { weight loss (FOWL) }\end{array}$} & 1 & 36.94 & 75.96 & 68.40 & 7.39 & 219.0 & 0,000 \\
\hline & II & 25.86 & 79.80 & 61.22 & 11.13 & 274.0 & 0,009 \\
\hline & III & 31.30 & 78.81 & 61.33 & 12.48 & 266.0 & 0,235 \\
\hline \multirow{3}{*}{$\begin{array}{l}\text { Egg laying } \\
\text { frequency (ELF) }\end{array}$} & 1 & \multicolumn{6}{|c|}{100} \\
\hline & II & \multicolumn{6}{|c|}{100} \\
\hline & III & \multicolumn{6}{|c|}{100} \\
\hline
\end{tabular}

$\mathrm{M}$ - arithmetic mean; SD - standard deviation

3) Egg Amount (EA). The number of deposited eggs decreased after the females had fed on a rabbit infested once and twice. The most abundant deposition yield (mean 1,637 \pm 154 eggs) was observed after infestation I, and the least abundant after infestations III (mean $872 \pm 117$ eggs) of the same host (Tab. $1,2)$. The observed differences appeared to be statistically significant for infestations I and II, as well as II and III, and I and III (in each case $\mathrm{U}=0.00 ; \mathrm{p}=0.049$ ). The egg amounts obtained in inter-specific groups were higher compared with the mono-specific groups. Statistical analysis confirmed the significance of these differences (Tab. 2).

Female oviposition weight loss (FOWL). The females feeding on a naive host (first infestation) were characterised by the largest percentage of body weight loss during the oviposition

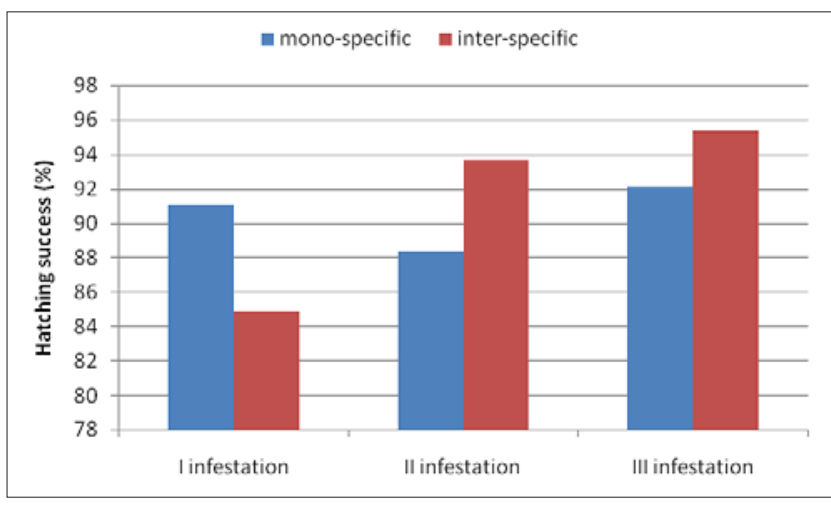

Figure 1. Hatching success in Ixodes ricinus after feeding on rabbit in species monospecific group and after co-feeding with Dermacentor reticulatus in 3 infestations

period (mean $68.40 \pm 7.39 \%$ ). The mean FOWL values in the re-infestations amounted to $61.22 \pm 11.13 \%$ after infestation II and $61.33 \pm 12.48 \%$ after infestation III. The weight loss during the oviposition period displayed statistical differences in infestations I and II, as well as I and III (Tab. 2). The values of the Mann-Whitney U test for infestations I and II and for I and III were $U=224.00 ; p=0.000$, and $U=273.00 ; p=0.008$, respectively. In comparison with the mono-specific group, the females feeding in the inter-specific groups utilized a higher percentage of body weight for egg production (Tab. 1).

Egg laying frequency (ELF). In the inter-specific groups, all the females in the 3 successive infestations ceased feeding and laid eggs; accordingly, the egg laying frequency was $100 \%$. Differences were only observed in the mono-specific group after infestation III, in which some females did not lay eggs after cessation of feeding (Tab. 1, 2).

Hatching success (HS). In infestation I, larvae hatched from the mean $84.85 \pm 1.00 \%$ of the eggs laid by the females. The mean HS values were similar in all the infestations; they reached $93.68 \pm 1.25 \%$ and $95.33 \pm 4.93 \%$ in infestations II and III, respectively, and did not differ statistically ( $p>0.05)$ (Fig. 1). Compared with the mono-specific group, the HS values in the inter-specific group were higher and differed significantly after infestations I and II ( $U=0.0 ; p=0,049$ and $\mathrm{U}=0.0 ; \mathrm{p}=0,049$ respectively).

\section{DISCUSSION}

Intra- and interspecies interactions are interesting for specialists in numerous scientific disciplines. They are especially significant when they concern parasites and their hosts. This is related mainly to the serious direct effects exerted by the parasites living in the host's internal organs or on the skin, or to the participation of some parasites in pathogen transmission. In the light of recent reports on the harmfulness of ticks, recognition of the interactions between these arthropods and their hosts, and between various tick species during infestation of the same host, presents a challenge to researchers and practitioners. The presented study on the commonest species in Europe - I. ricinus and D. reticulatus - has demonstrated the biological consequences of the interactions, both for the parasites and their hosts. These data can be used for elaborating methods of prevention of tick infestations and the effects of parasitism. Knowledge of the 
mutual 2-species interactions on the host may contribute to explication of the mechanisms of harmful effects of parasites' activity and various ways of pathogen transmission, e.g. during co-feeding and single-species feeding.

The presented study, the first worldwide, demonstrates the mutual influence exerted by 2 tick species: $I$. ricinus and $D$. reticulatus on the efficiency of biological processes in these ticks.

Tick antigens introduced the host induce innate and acquired immune response [10-12]. Predominance of one of the immunity types depends on numerous factors, mainly on the tick species and its developmental stage, the length of the feeding period, number of feeding ticks, number of infestations on hosts, and on the individual sensitivity of the host.

Barriga et al. [13] demonstrated that ticks stimulate the host's immune system to produce immune cells specific for over 40 antigens contained in the salivary gland secretion. Throughout the feeding period, salivary glands produce substances that have suppressive activity practically in each phase of the innate and acquired immune response $[14,15]$.

According to Barriga et al. [13], potent immunogenic antigens appearing in the second phase of feeding are likely to 'distract' the immune system from the indispensable for blood uptake proteins injected to the host's skin by a tick, which are markedly less potent allergens than other compounds of salivary secretion. They thus exert an immunosuppressive effect on the host immune system, and provide the tick with the necessary quality and quantity of food throughout the feeding period.

Salivary gland allergens are detected in the host's skin within a week after feeding cessation; therefore, host immunisation is prolonged [16]. Tick antigens stimulate immune cells in the skin - macrophages, mastocytes and basophils - to produce pro-inflammatory cytokine (e.g., IL-1, IL-3, IL-5, IL-6, IL-6, IL-8, TNF- $\alpha$ ), and immune factors. However, some compounds secreted by ticks inhibit migration of these cells to the feeding sites of these arthropods.

A decrease in the TNF- $\alpha$ plasma level in rabbits' blood was observed under feeding conditions that were optimal for $D$. reticulatus ticks. Since TNF- $\alpha$ enhances immune cell proliferation and differentiation, activates eosinophil cytotoxicity and is an important mechanism of anti-infectious response, a decrease in its level ensures effective feeding and facilitates pathogen transmission into the host organism. The increase in its concentration in the final feeding phase, observed in the current study, is a consequence of the immune system activation connected with the developing host resistance to the arthropod infestation (unpublished data).

All the I. ricinus and D. reticulatus females in the monospecific and inter-specific infestations fed on the rabbit. The tick yield, i.e. the number of $I$. ricinus females which ceased feeding with the body mass at least $0.1 \mathrm{~g}$ per number of all engorged females, reached $100 \%$ in infestations I and II. The tick yield decreased only in mono-specific and inter-specific infestation III to reach $80 \%$ and $97 \%$, respectively, due to the acquired host resistance. The tick yield in D. reticulatus females reached $100 \%$ in both experiments.

A statistically significant increase in the I. ricinus body weight was observed during co-feeding with $D$. reticulatus in both infestations I and III. Compared with the mono-specific groups, the I. ricinus egg amount in the inter-specific groups in the 3 infestations increased significantly, but the egg mass weight was not altered in a significant manner.

The $I$. ricinus feeding sites displayed numerous migratory cells with degradation and metabolism disturbances, fibroblasts with enhanced metabolic activity, debris of damaged fibroblasts with signs of the initial necrosis phase, accumulation of mono-specific protein secretion -a sign of interstitial oedema, and vessels with necrotic endothelial cells [17]. In the feeding site of D. reticulatus, the lesions were less pronounced; there were migratory cells with a varied degree of activation, fibroblasts with cell damage traits, interstitial oedema, and fragmented collagen fibres. The skin capillary vessels had a normal structure accompanied by extravascular exudate $[17,18]$.

More pronounced lesions at the feeding site appeared on the host skin after tick re-infestations; basophil granulocytes were predominant and, together with mast cells, released anaphylactic reaction mediators and chemotactic factors which participate in activation of the immune system. This process is effectively inhibited by substances present in the saliva, e.g., the C3a component of the complement system (inhibition of the complement system) $[15,19]$ and apyrases (which block the release of pro-inflammatory mediators from mastocytes by conversion of ATP to ADP).

In subsequent $D$. reticulatus infestations, a bigger range and enhanced intensity of skin lesions in the host was observed. As early as during the infestation there were purulent-necrotic centres in the dermis. The infiltrations contained mainly cells with one nucleus and included, to name a few, mastocytes, plasmocytes, lymphocytes, and fibroblasts with numerous neutrophil granulocytes and eosinophil granulocytes, which penetrated inside the collagen fibre bundles. Large necrotic areas were observed in the dermis. In the secondary infestations, purulent lesions were visible in the hypodermis, subcutaneous adipose layer and the muscularis mucosae (unpublished data).

Feeding on a non-immunised host by $I$. ricinus females produced an approx. $1.54 \mathrm{~mm}^{2}$ inflammatory zone which extended to the hypodermis, but did not cross the skin-muscle border. During re-infestation, the area of the inflammatory zone enlarged and reached $5.91 \mathrm{~mm}^{2}$ and the large purulent lesions reached the muscle layer [20].

Data obtained by Szabo and Bechara [21] suggest that in repeated tick infestations, basophils and eosinophils are the cells involved in resistance of the guinea pigs to Rhipicephalus sanguineus.

The prolonged feeding period in re-infestations may have been caused by disturbances in the food intake mechanism. The feeding process involves alternate saliva pumping due to contraction of the pharyngeal muscles and sucking the food composed of blood, interstitial fluid, dissolved tissues and injected saliva. The biggest amount of food is ingested in the second phase of feeding [22]. During feeding, serum components enter the midgut and haemolymph [23-27]. High titres of guinea-pig IgG antibodies were found in the saliva of both partially fed Rhipicephalus appendiculatus females and after 6 days of the feeding period [28, 29].

The observations in the presented study and reports by other authors indicate that subsequent infestations induce host resistance. Although acquired resistance to tick feeding is species specific, examples of cross-resistance to infestations with different tick species are well-known [30-35]. Cross-resistance appeared between Metastriata 
species, i.e. R. appendiculatus and Amblyomma variegatum [35], Hyalomma anatolicum anatolicum and Rhipicephalus evertsi [31] Hyalomma anatolicum anatolicum and Boophilus microplus [36], A. americanum and D. variabilis [30]. Feeding on rabbits resistant to $D$. andersoni resulted in a reduced increase in body mass in I. scapularis [30].

In natural conditions, $I$. ricinus and $D$. reticulatus are active in the same periods, and therefore concurrently feed on a host. Nevertheless, secondary infestations in natural conditions reduce the tick population abundance in nature by the effect exerted on female reproduction.

In other studies by the authors, the biggest disturbances in tick development were observed when the secondary infestations occurred 21 days after the first, i.e. in the period of enhanced resistance of the host. The increased antibody level in the host after several days after the beginning of the primary infestation was probably the cause of greater host's resistance in secondary infestations occurring after a long term (unpublished data).

During repeated infestation with other tick species, lowered efficiency of some physiological processes, first of all of feeding, maturation and oviposition processes [34, 37-41], embryonic development, as well as larval hatching and molting of the subsequent developmental stages [42-44] were observed. Moreover, lethality of the active tick stages was increased (unpublished data).

In a previous study by Schorderet and Brossard, on the effect of rabbit's immunisation on I. ricinus [45], they demonstrated an increasing number of attached ticks, decreased amounts of ingested food, extension and disturbances in the feeding period, disturbances in the maturation and oviposition processes and in the development of the subsequent developmental stages upon infestations that took place at 25day long intervals. Similar dependencies were also observed in another study of $D$. reticulatus feeding on the rabbit in mono-specific groups (unpublished data). Some differences between the results obtained by the above-mentioned authors and the results of the present study (the percentage of normally hatched larvae) stem from the different degree of host immunisation (different re-infestation time) and from different methodologies employed (different experimental conditions). In I. scapularis feeding on immunised dogs, oviposition disturbances and decreased larval survival in the first generation were observed [46]. The current results suggest that co-feeding 2 different tick species on the same host may partially counteract the adverse influence of host immunity in repeated invasions.

The results of the presented study and observations by other authors demonstrate the high complexity of interactions between individuals of the same species, and between ticks from different species feeding on one host and the tick-host interactions. The diverse mechanisms of these interactions have not yet been explored. Their elucidation requires further biological, chemical and immunological investigations.

\section{REFERENCES}

1. Kahl O, Janetzki C, Gray JS, Stein J, Bauch RJ. Tick infection rates with Borrelia: Ixodes ricinus versus Haemaphysalis concinna and Dermacentor reticulatus in two locations in eastern Germany. Med Vet Entomol. 1992; 6(4): 363-366.

2. Stanko M, Miklasova D. Infestation trends of two rodent species (Rodentia, Muridae) on the East Slovakian lowland. In: Buczek A,
Błaszak C, editors. Arthropods in Medicine. Lublin: Liber. 2002: 105-114.

3. Földvári G, Farkas R. Ixodid tick species attaching to dogs in Hungary. Vet Parasitol. 2005; 129(1-2): 125-131.

4. Bursali A, Keskin A, Tekin S. Ticks (Acari: Ixodida) infesting humans in the provinces of Kelkit Valley, a Crimean-Congo Hemorrhagic Fever endemic region in Turkey. Exp Appl Acarol. 2013; 59(4): 507-515.

5. Gern L, Rais O. Efficient transmission of Borrelia burgdorferi between cofeeding Ixodes ricinus ticks (Acari: Ixodidae). J Med Entomol. 1996; 33(1): 189-192.

6. Labuda M, Alves MJ, Eleckova E, Kozuch O, Filipe AR. Transmission of tick-borne bunyaviruses by cofeeding ixodid ticks. Acta Virol. 1997; 41(6): 325-328.

7. Buczek A, Bartosik K, Zając Z, Stanko M. Host-feeding behaviour of Dermacentor reticulatus and Dermacentor marginatus in mono-specific and inter-specific infestations. Parasit Vectors. 2015; 8: 470.

8. Bowessidjaou J, Brossard M, Aeschlimann A. Effects and duration of resistance acquired by rabbits on feeding and egg laying in Ixodes ricinus L. Cell Mol Life Sci. 1977; 33(4): 528-530.

9. Jittapalapong S, Stich RW, Gordon JC, Wittum TE, Barriga OO. Performance of female Rhipicephalus sanguineus (Acari: Ixodidae) fed on dogs exposed to multiple infestations of immunization with tick salivary gland or midgut tissues. J Med Entomol. 2000; 37(4): 601-611.

10. Papatheodorou V, Brossard M. C3 levels in the sera of rabbits infested and reinfested with Ixodes ricinus L. and in midguts of fed ticks. Exp Appl Acarol. 1987; 3(1): 53-59.

11. Brossard M, Wikel SK. Immunology of interactions between ticks and hosts. Med Vet Entomol. 1997; 11(3): 270-276.

12. Willadsen P, Jongejan F. Immunology of the tick-host interaction and the control of ticks and tick-borne diseases. Parasitol Today. 1999; 15(7): 258-262.

13. Barriga OO, Andujar F, Andrzejewski WJ. Manifestations of immunity in sheep repeatedly infested with Amblyomma americanum ticks. J Parasitol. 1991; 77(5): 703-709.

14. Ribeiro JM, Makoul GT, Levine J, Robinson DR, Spielman A. Antihemostatic, antiinflammatory, and immunosuppressive properties of the saliva of a tick, Ixodes dammini. J Exp Med. 1985; 161(2): 332-344.

15. Wikel SK. Tick modulation of host immunity: an important factor in pathogen transmission. Int J Parasitol. 1999; 29(6): 851-859.

16. Allen J R, Khalil HM, Graham JE. The location of salivary antigens, complement and immunoglobulin in the skin of guinea-pigsinfested with Dermacentor andersoni larvae. Immunology 1979; 38(3): 467-472.

17. Buczek A, Kuśmierz A, Olszewski K, Buczek L, Czerny K, Łańcut M. 2002. Comparsion of rabbit skin changes after feeding of Ixodes ricinus (L.) and Dermacentor reticulatus (Fabr.). In: Bernini F, Nannelli R, Nuzzaci G, de Lillo E, editors. Acarid Phylogeny and Evolution: Adaptation in Mites and ticks. Dordrecht: Kulwer Academic Publisher. 2002; 419-424.

18. Olszewski K, Kuśmierz A, Buczek L, Buczek A. Skin chan ges caused by feeding of Ixodes ricinus (Linneaus, 1758) and Dermacentor reticulatus (Fabricius, 1794) in the electron microscope. In: Buczek A, Błaszak C, editors. Arthropods in Medicine. Lublin: Liber. 2002; 197-203 (in Polish).

19. Ribeiro JM, Spielman A. Ixodes dammini: salivary anaphylatoxin inactivating activity. Exp Parasitol. 1986; 62(2): 45-51.

20. Buczek A, Buczek L, Kuśmierz A, Olszewski K. Skin changes in rabbit skin caused by ticks (Acari: Ixodida). In: Buczek A, Błaszak C, editors. Parasitic and Alergogenic Arthropods. Lublin: KGM. 2000; 171-182 (in Polish).

21. Szabo MPJ, Bechara GH. Sequential histopathology at the Rhipicephalus sanguineus tick feeding site on dogs and guinea pigs. Exp Appl Acarol. 1999; 23(11): 915-928.

22. Gregson JD. Observations on the movement of fluids in the vicinity of the mouthparts of naturally feeding Dermacentor andersoni Stiles. Parasitology 1967; 57(1): 1-8.

23. Ackerman S, Claire FB, McGill TW, Sonenshine DE. Passage of host serum components, including antibody, across digestive tract of Dermacentor variabilis (Say). J Parasitol. 1981; 67(5): 737-740.

24. Brossard M, Rais O. Passage of haemolysing through the midgut epithelium of female Ixodes ricinus L. fed on rabbits infected or reinfected with ticks. Experientia. 1984; 40: 561-562.

25. Fujisaki K, Kamio T, Kitaoka S. Passage of host serum components, including anti-bodies specific for Theileria sergenti, across the digestive tract of argasid and ixodid ticks. Ann Trop Med Parasitol. 1984; 78(4): 449-450.

26. Ben-Yakir D. Quantitative studies of host immunoglobulin G in the hemolymph of ticks (Acari). J Med Entomol. 1989; 26(4): 243-246. 
27. Need JT, Butler JF. Possible application of the immune response of laboratory mice to the feeding of argasid ticks. J Med Entomol. 1991; 28(2): 250-253.

28. Wang H, Nuttall PA. Extraction of host immunoglobulin in the saliva and detection of igG- binding protein in tick hemolymph and salivary gland. Parasitology 1994; 109(4): 525-530.

29. Wang H, Nuttall PA. Comparison of the proteins in salivary gland, saliva, and haemolymph of Rhipicephalus appendiculatus female ticks during feeding. Parasitology 1994; 109(4): 517-523.

30. McTier T, George J, Bennett S. Resistance and cross-resistance of guinea pigs to Dermacentor andersoni Stiles, D. variabilis (Say), Amblyomma americanum (Linnaeus), and Ixodes scapularis Say. J Parasitol. 1981; 67(6): 813-822.

31. Latif AA. Resistance to Hyalomma anatolicum anatolicum Koch (1844) and Rhipicephalus evertsi Neumann (1897) (Ixodoidea: Ixodidae) by cattle in the Sudan. Insect Sci Appl. 1984; 5(6): 509-511.

32. Allen JR. Immunology of interactions between ticks and laboratory animals. Exp Appl Acarol. 1989; 7(1): 5-13.

33. De Castro JJ, Newson RM, Herbert IV. Resistance in cattle against Rhipicephalus appendiculatus with an assessment of cross-resistance to R. pulchellus (Acari: Ixodidae). Exp Appl Acarol. 1989; 6(3): 237-244

34. Rechav Y, Heller-Haupt A, Varma MG. Resistance and cross-resistance in guinea pigs and rabbits to immature stages of ixodid ticks. Med Vet Entomol. 1989; 3(4): 333-336.

35. Heller-Haupt A, Kagaruki LK, Varma MGR. Resistance and crossresistance in rabbits to adults of three species of African ticks (Acari: Ixodidae). Exp Appl Acarol. 1996; 20(3): 155-165.

36. Parmar A, Grewal AS, Dhillon P. Immunological crossreactivity between salivary gland proteins of Hyalomma anatolicum and Boophilus microplus ticks. Vet Immunol Immunop. 1996; 51(3-4): 345-352.
37. Musatov VA. Changes in physiology and size of ixodid ticks when feeding repeatedly on the same animal. Parazitologiya 1967; 1(4): 288-292.

38. Allen JR. Tick resistance: basophils in skin reactions of resistant guinea pigs. Int J Parasitol. 1973; 3(2): 195-200.

39. Rahman MH. Effects of host-immunity on the life cycle of Hyalomma rufipes Koch, 1844 (Ixodida: Ixodidae). Folia Parasitol (Praha) 1974; 31(1): 63-67.

40. Brossard M, Girardin P. Passive transfer of resistance in rabbits infested with adult Ixodes ricinus L.: humoral factor influence feeding and egg laying. Experientia 1979; 35(10): 1395-1397.

41. Wikel SK. Host immunity to ticks. Ann Rev Entomol. 1996; 41: 1-22.

42. Latif AA, Newson RM, Dhadialla TS. Feeding performance of Amblyomma variegatum (Acarina: Ixodidae) fed repeatedly on rabbits. Exp Appl Acarol. 1988; 5(1-2): 83-92.

43. Linthicum KJ, Dickson DL, Logan TM. Feeding efficiency of larval Hyalomma truncatum (Acari) on hosts previously exposed to ticks. J Med Entomol. 1992; 29(2): 310-313.

44. Bagnall BG, Rothwell TLW. Responses in guinea pigs to larvae of tick, Ixodes holocyclus. In: Proceedings $3^{\text {rd }}$ Inter Congr Parasitol Facta Publ: 1974; 2: 1082-1083.

45. Schorderet S, Brossard M. Changes in immunity to Ixodes ricinus by rabbits infested at different levels. Med Vet Entomol. 1993; 7(2): 186-192.

46. Gebbia JA, Bosler EM, Evans RD, Schneider EM. Acquired resistance in dogs to repeated infestation with Ixodes scapularis (Acari: Ixodidae) reduces tick viability and reproductive success. Exp Appl Acarol. 1995; 19(10): 593-605. 